\title{
Une surface de Voss à 6 dimensions dont le sous-espace osculateur est à 9 dimensions.
}

\author{
Memoria di S. Finikor
}

A M. Enrico Bompiani pour un Jubilé scieutificue.

§1. Considérons une surface $\mathscr{\Upsilon K}_{6}$ plongée dans l'espace euclidien $E_{n}$, qui porte un réseau conjugué, composé de six familles des lignes géodésiques. Supposons que le réseau en question est holonome, c.à.d. admet une transformation de LAPLACE pour chaque couple de familles conjuguées de lignes géodésiques.

Si les vecteurs unitaires $\overrightarrow{e_{i}}(i=1, \ldots 6)$ de longueur $1 \mathrm{du}$ repère attaché au point $M$ de $\mathfrak{T K}_{6}$ touchent des lignes géodésiques du réseau de Voss, tandis que les vecteurs $\vec{e}_{\alpha}(\alpha=7,8,9)$ avec les $\overrightarrow{e_{i}}$ déterminent le sousespace osculateur $E_{9}$, les premiéres équations dérivatives de $\mathfrak{T K}_{6}$ s'écrivent:

$$
\begin{array}{ll}
d \vec{M}=\omega^{r} \overrightarrow{e_{i}} & (i, j=1, \ldots 6) \\
\overrightarrow{d \overrightarrow{e_{i}}}=\omega_{i}^{\overrightarrow{e_{j}}}+\omega_{i}^{\alpha \overrightarrow{e_{\alpha}}} & (\alpha, \beta=7,8,9) \quad(1: 1) \\
\overrightarrow{d \overrightarrow{e_{\alpha}}}=\omega_{\alpha}^{1} \overrightarrow{e_{j}}+\omega_{\alpha}^{\beta e_{\beta}}+\omega_{\alpha}^{\lambda} \overrightarrow{e_{\lambda}} ; & (\lambda=10, \ldots)
\end{array}
$$

les formes $\omega^{t}, \omega_{i}^{i}, \omega_{\alpha}^{\beta}$ etc. vérifient les équations de la structure euclidienne; les vecteurs $\vec{e}_{\lambda}$ avec les précédents déterminent le second sous-espace osculateur.

La différenciation extérieure de l'identité évidente

$$
\omega^{\alpha}=0
$$

nous donne

$$
\left[\omega^{i} \omega_{i}^{\alpha}\right]=0
$$

d'où à l'aide du lemme de CARTaN on obtient

$$
\omega_{i}^{\alpha}=l_{i j}^{\alpha} \omega^{\jmath} \quad l_{i j}^{\alpha}=l_{j i}^{\alpha}
$$


et les formes quadratiques asymptotiques sont $\overrightarrow{e_{\alpha}} d^{2} \vec{M}=\overrightarrow{e_{\alpha}}, d\left(\omega \overrightarrow{e_{i}}\right)=\omega^{i} \overrightarrow{e_{\alpha}} \overrightarrow{d_{i}}=$ $=\omega^{i} \omega_{i}^{\beta e_{\alpha}} \overrightarrow{e_{\beta}} ;$ remplaçons maintenant le $\omega_{i}^{\beta}$ par son valeur $(1: 2)$ et posons

$$
\overrightarrow{e_{\alpha}} \overrightarrow{e_{\beta}}=\rho_{\alpha \beta} . \quad \operatorname{det}\left|\rho_{\alpha \beta}\right| \neq 0
$$

nous obtenons

$$
\Phi_{\alpha}=\rho_{\alpha \beta} l_{i j}^{\beta} \omega^{\prime} \omega^{j}
$$

Deux familles de lignes

$$
\omega^{a}\left(d_{1}\right)=\delta_{1}^{a}, \quad \omega^{a}\left(d_{2}\right)=\delta_{2}^{a} \quad \delta_{b}^{a}=\left\{\begin{array}{l}
0, \text { si } \alpha \pm b \\
1, \text { si } \alpha=b
\end{array}\right.
$$

sont conjugués, si la forme bilinéaire

$$
\Phi_{\alpha}\left(d_{1}, d_{2}\right)=\rho_{\alpha \beta} l_{i j}^{\beta} \omega^{t}\left(d_{1}\right) \omega^{j}\left(d_{2}\right)=0
$$

est nulle. En y portant les valeurs $(1: 5)$, on obtient

$$
l_{12}^{\beta}=0
$$

En général la condition que chaque couple de lignes $i, j=1, \ldots, 6$ soit conjugué est l'annulation de tous les coefficients

$$
l_{i j}^{\beta}=0, \quad(i \neq j
$$

à deux indices en bas différents.

$$
\Phi_{\alpha}=\sum_{\substack{i=1, \ldots \ldots 6 \\ \beta=7, \mathrm{~s}_{9}^{6}}} p_{\alpha \beta} l_{i i}^{\beta}\left(\omega^{t}\right)^{2}
$$

Or, le sous-espace $E_{9}$ non dégénéré, ces formes sont linéairement independantes.

En les remplaçant par leur combinaison linéaires, on réduit à la façon

$$
\Psi^{i+6}=l_{i i}^{i+6}\left(\omega^{i}\right)^{2}+\sum_{j=4,5,6} l_{j i}^{i+6}\left(\omega^{j}\right)^{2} \quad(i=1,2,3)
$$


Supposons, que la surface a le système des formes quadratiques

$$
l_{11}^{7}\left(\omega^{1}\right)^{2}+l_{44}^{7}\left(\omega^{4}\right)^{2}+l_{66}^{7}\left(\omega^{6}\right)^{2}
$$

$$
\begin{aligned}
& l_{22}^{8}\left(\omega^{2}\right)^{2}+l_{55}^{8}\left(\omega^{5}\right)^{2} \\
& l_{33}^{9}\left(\omega^{3}\right)^{2}
\end{aligned}
$$

et les composants $\omega_{i}^{\alpha}$ par le choix convenable des vecteurs $\overrightarrow{e_{\alpha}}$ se réduisent à la forme

$$
\omega_{1}^{7}=\lambda_{1} \omega^{1}, \omega_{4}^{7}=\lambda_{4} \omega^{4}, \omega_{6}^{7}=\lambda_{6} \omega^{6},
$$

$$
\begin{aligned}
& \omega_{2}^{8}=\lambda_{2} \omega^{2}, \quad \omega_{5}^{8}=\lambda_{5} \omega^{5}, \\
& \omega_{3}^{9}=\lambda_{3} \omega^{3}
\end{aligned}
$$

et toutes les autres sont égales à zéro.

$$
\omega_{2}^{7}=\omega_{3}^{7}=\omega_{5}^{7}=0
$$

$$
\begin{aligned}
& \omega_{1}^{8}=\omega_{3}^{8}=\omega_{4}^{8}=\omega_{6}^{8}=0, \\
& \omega_{1}^{9}=\omega_{2}^{9}=\omega_{4}^{9}=\omega_{5}^{9}=\omega_{6}^{9}=0 .
\end{aligned}
$$

Admettons que tous les coefficients $\lambda_{i}$ sont différents de zéro.

§ 2. De même, si l'on différencie l'identité évidente

$$
\omega_{i}^{\lambda}=0
$$

on obtient

$$
\left[\omega_{i}^{\alpha} \omega_{\alpha}^{\lambda}\right]=0
$$

en vertu de $(1: 10),(1: 11)$ les équations prennent la forme

(2:1)

$$
\begin{aligned}
& {\left[\omega^{1} \omega_{7}^{\lambda}\right]=0, \quad\left[\omega^{2} \omega_{8}^{\lambda}\right]=0, \quad\left[\omega^{3} \omega_{9}^{\lambda}\right]=0,} \\
& {\left[\omega^{4} \omega_{7}^{\lambda}\right]=0, \quad\left[\omega^{5} \omega_{8}^{\lambda}\right]=0} \\
& {\left[\omega^{6} \omega_{7}^{\lambda}\right]=0}
\end{aligned}
$$


Trois équations de la premiére colonne en vertu du lemme de CarTaN exigent proportionalité de la forme $\omega_{7}^{2}$ aux formes $\omega^{ \pm}, \omega^{4}, \omega^{6}$ à la fois.

Or les formes $\omega^{t}$ sont linéairement indépendantes, donc la forme $\omega_{7}^{2}$ s'annule; de même la forme $\omega_{8}^{\lambda}$

$$
\omega_{7}^{\lambda}=0, \quad \omega_{8}^{\lambda}=0, \quad \omega_{9}^{\lambda}=b_{9}^{\lambda} \omega^{9},
$$

les équations (1:1) de la dernière ligne prennent maintenant la forme

$$
\overrightarrow{d e_{7}}=\omega_{7}^{j} \overrightarrow{e_{j}}+\omega_{7}^{\vec{\beta} e_{\beta}}
$$

$$
\begin{aligned}
& \overrightarrow{d \overrightarrow{e_{8}}}=\omega_{8}^{i \overrightarrow{e_{j}}}+\omega_{8}^{\beta} \overrightarrow{e_{\beta}}, \\
& \overrightarrow{d \overrightarrow{e_{9}}}=\omega_{9}^{j} \overrightarrow{e_{j}}+\omega_{9}^{\beta} \overrightarrow{e_{\beta}}+a_{9}^{10} \overrightarrow{\omega^{3} e_{10}},
\end{aligned}
$$

où le vecteur $e_{10}$ de longueur 1 est déterminé par l'équation

$$
a_{9}^{10} \overrightarrow{e_{10}}=b_{8}^{\lambda} \overrightarrow{e_{\lambda}}
$$

et

$$
\omega_{7}^{10}=0, \quad \omega_{8}^{10}=0, \quad \omega_{9}^{10}=a_{9}^{10} \omega^{3} .
$$

Ainsi la dimension du second sous-espace osculateur $E_{10}$ est plus grande d' une unité que celle du premier.

§ 3. Deux familles de lignes admettent une transformation de LAPLACE, si les tangentes aux lignes de la première famille engendrent une surface développable, quand le point de contact décrit une ligne de la seconde famille, c.a.d. s' il existe un point

$$
\vec{M}_{1}=\vec{M}+\rho \vec{e}_{k_{1}}
$$

sur la tangente $\overrightarrow{e_{k 1}}$, dont le déplacement infinitésimal

$$
d_{2} \vec{M}_{1}=d_{2}\left(\vec{M}+\rho \overrightarrow{e_{k}}\right)=
$$

$$
=\omega^{k_{2}} \vec{e}_{k_{2}}+\vec{e}_{k_{1}} d_{2 \rho}+\rho \sum_{j} \omega_{k_{1}}^{i}\left(d_{2}\right) \vec{e}_{j}
$$

coincide avec la direction de la tangente $\overrightarrow{\boldsymbol{e}}_{k_{1}}$.

Fn posant

$$
\omega_{k_{1}}^{j}=\sum_{i} l_{k_{1} i}^{j} \omega^{i}
$$


done

$$
\omega{ }_{k_{1}}^{i}\left(d_{2}\right)=l_{k_{1} k_{2}}^{j} \omega^{k_{2}}
$$

en omettant les termes contenant le vecteur $\vec{e}_{k_{1}}$, on reçoit

$$
\omega \vec{k}^{k} \overrightarrow{e_{k_{2}}}+\rho \sum_{j \neq k_{1}} l_{k_{1} k_{2}}^{j} \omega^{k_{2}} \overrightarrow{e_{j}}=0
$$

d' où en égalant les coefficients des vecteurs ${\overrightarrow{e_{k}}}_{2}, \overrightarrow{e_{j}}\left(j \neq K_{1}, K_{2}\right)$ on obtient

$$
1+\rho l_{k_{1} k_{2}}^{k_{z_{2}}}=0, \quad l_{k_{1} k_{2}}^{j}=0 . \quad\left(j \neq k_{1} k_{2}\right)
$$

La premiére de ces èquations détermine $\rho$, c.a.d. la position du foyer $M_{1}$ sur la droite $\left(M ; \vec{e}_{k_{1}}\right)$; la seconde restreint les formes $\omega_{k}^{j}$ : les coef. ficients $l_{k_{1} k_{2}}^{j}$ à trois indices différents s' annulent.

L'équation (3:2) prend la forme

$$
\omega_{i}^{j}=l_{i i}^{i} \omega^{i}+l_{i j}^{i} \omega^{j}
$$

Si en outre, les lignes coordonnées sont géodésiques, c.a.d. chaque vecteur $e_{i}$ au déplacement $d_{,}\left(\omega^{k}={ }_{i}^{k}\right)$ ne tourne pas dans le sous-espace tangent, le coefficient $l_{i i}^{i}$ est nul et l'égalité $(3: 3)$ devient

$$
\omega_{i}^{i}=l_{i i}^{i} \omega^{i}
$$

§ 4. Les équations $(1: 10),(1: 11),(3: 4)$ forment le système différentiel du problème. Elles ont beaucoup de consequences différentielles.

En dérivant exterieurement les équations (1: 11), on obtient en vertu de $(1: 10),(3: 4)$

$$
\left\{\begin{array}{l}
{\left[\omega_{7}^{8} \omega^{1}\right]=0, \quad\left[\omega_{7}^{9} \omega^{1}\right]=0, \quad\left[\omega_{8}^{9} \omega^{1}\right]=0, \quad\left[\omega_{8}^{7} \omega^{2}\right]=0} \\
{\left[\omega_{7}^{8} \omega^{4}=0, \quad\left[\omega_{7}^{9} \omega^{4}\right]=0, \quad\left[\omega_{8}^{9} \omega^{3}\right]=0, \quad\left[\omega_{8}^{7} \omega^{5}\right]=0\right.} \\
{\left[\omega_{7}^{8} \omega^{6}\right]=0, \quad\left[\omega_{7}^{9} \omega^{6}\right]=0,}
\end{array}\right.
$$

$$
\left[\omega_{9}^{7} \omega^{3}\right]=0, \quad\left[\omega_{9}^{8} \omega^{3}\right]=0 .
$$

En traitant comme le système (2:1), on obtient

$$
\begin{gathered}
\omega_{7}^{8}=0, \quad \omega_{8}^{7}=0, \quad \omega_{7}^{9}=0, \quad \omega_{8}^{9}=0, \\
\omega_{9}^{7}=a_{9}^{7} \omega^{3}, \quad \omega_{9}^{8}=a_{9}^{8} \omega^{3} .
\end{gathered}
$$


Or, on peut poser

$$
\overrightarrow{e_{7}} \overrightarrow{e_{10}}=\overrightarrow{e_{8}} \overrightarrow{e_{10}}=\overrightarrow{e_{9}} \vec{e}_{10}=0
$$

En différenciant ces identités, on obtient

$$
\omega_{10}^{7}+\omega_{10}^{8} \rho_{78}+\omega_{10}^{9} \rho_{79}=0
$$

$$
\begin{aligned}
& m_{10}^{7} \rho_{87}+\omega_{10}^{8}+\omega_{10}^{9} \rho_{89}=0 \\
& \omega_{10}^{7} \rho_{97}+\omega_{10}^{8} \rho_{98}+\omega_{10}^{9}=-\omega_{9}^{10}=-a_{9}^{10} \omega^{3} .
\end{aligned}
$$

Si l'on multiplie les équations (4:5) successivement par les mineurs réduites $\pi^{\alpha \beta}$ du déterminant $(1: 3)$ et prend la somme, on obtient

$$
\omega_{10}^{7}=-\pi^{79} a_{9}^{10} \omega^{3}, \omega_{10}^{8}=-\pi^{89} a_{9}^{10} \omega^{3}, \omega_{10}^{9}=-a_{9}^{10} \omega^{3}
$$

De mềme, en différenciant les identités

$$
\left(\vec{e}_{7}\right)^{2}=\left(\vec{e}_{8}\right)^{2}=\left(\vec{e}_{9}\right)=\left(\vec{e}_{10}\right)^{2}=1
$$

on obtient

$$
\begin{gathered}
\omega_{7}^{7}=\omega_{8}^{8}=0, \quad \omega_{10}^{10}=0_{1} \\
\omega_{9}^{9}=-\left(a_{9}^{7} \rho_{97}+a_{9}^{8} \rho_{98}\right) \omega^{3}=a_{9}^{9} \omega^{3},
\end{gathered}
$$

si l'on pose

$$
a_{9}^{7} \rho^{97}+a_{9}^{8} \rho^{98}+a_{9}^{9}=0
$$

§. En differénciant extérieurement les équations $(1: 10)$ on obtient

$$
\left(I_{1-6}\right) \quad\left[\Delta \lambda_{i} \omega^{i}\right]=0
$$

où

$$
\Delta \lambda_{k}=d \lambda_{k}-2 \lambda_{k} \omega_{k}^{k}+\lambda_{k} \sum_{j \neq k}^{j=1, \ldots, 6} l_{j k}^{k} \omega^{j}, \quad(k=1,2,4,5,6)
$$

$$
\Delta \lambda_{3}=d \lambda_{3}-2 \lambda_{3} \omega_{3}^{3}-a_{9}^{9} \lambda_{3} \omega^{3}+\lambda_{3} \sum_{i \neq 3}^{j=1 \ldots 6} l_{j 3}^{3} \omega^{j}
$$


S. Finikor : Une surface de Voss à 6 dimensions dont le sous-espace, etc. $\quad 47$

En différenciant extérieurement les equations (3:4), on obtient 30 équations, q' on pent écrire en trois groupes numérotés avec deux indices $(5: i, j)$, où $i, j=1, \ldots, 6, i \neq j$.

$$
\begin{aligned}
& {\left[\Delta l_{i j}^{j} \omega^{j}\right]+\lambda^{j}\left[\omega_{7}^{i} \omega^{i}\right]=0, \quad(i=1,4,5)} \\
& {\left[\Delta l_{i j}^{j} \omega^{j}\right]+\lambda_{i}\left[\omega_{3}^{j} \omega^{i}\right]=0, \quad(i=2,5)} \\
& {\left[\Delta l_{i j}^{j} \omega^{j}\right]+\lambda_{i}\left[\omega_{9}^{j} \omega^{i}\right]=0, \quad(i=3) .}
\end{aligned}
$$$$
(5: i, j)
$$

It est facile à voir que chaque forme $\omega_{7}^{j}$ entre an moins à deux équations $i=1,4,6 ; i \neq j$.

Or ces équations contiennent la même forme $\omega^{j}$, indépendante sur la variêté intégrale, et différentes fornes $\omega^{t}$; donc la forme $\omega^{i}$ ne dépend que de la forme $\omega^{i}$

$$
\omega_{7}^{j}=\omega_{7}^{j} \omega^{j} \quad(j=1, \ldots, 6)
$$

et les équations $(5: i, j)$ du premier groupe $(i=1,4,6)$ si l'on fait substitution (5:4), prennent la forme

$$
\left(\mathrm{II}_{1-15}\right)\left[\Delta l_{i j}^{i} \omega^{j}\right]=0\left(\begin{array}{l}
i=1,4,6 \\
j=1, \ldots, j \neq i
\end{array}\right)
$$

où

$$
\nabla l_{i j}^{i}=\Delta l_{i j}^{j}-\lambda_{i} a_{7}^{i} \omega^{\mathbf{i}}
$$

De mème, les équations $(5: i, i)$ du second groupe $(i=2,5)$ donnent

$$
\omega_{8}^{j}=a_{8}^{j} \omega^{j} \quad(j=1,3,4,6)
$$

et les équations quadratiques

$$
\left(\mathrm{II}_{16-24}\right) \quad\left[\nabla l_{2 j}^{j} \omega^{i}\right]=0,\left[\nabla l_{5 j}^{j} \omega^{j}\right]=0 \quad(j=1,3,4,6)
$$

où

$$
\nabla l_{2 j}^{j}=\Delta l_{2 j}^{i}-\lambda_{2} a_{8}^{j} \omega^{2}
$$

$$
\nabla l_{5 j}^{i}=\Delta^{l j}{ }_{5 j}^{j}-\lambda_{5} a_{8}^{j} \omega^{5} .
$$


Il nous reste deux équations du second groupe $(5: i, j)$

$$
\left(\mathrm{IV}_{1.2}\right) \quad\left[\Delta l_{25}^{5} \omega^{5}\right]+\lambda_{2}\left[\omega_{8}^{5} \omega^{2}\right]=0
$$

$$
\left[\Delta l_{52}^{2} \omega^{2}\right]+\lambda_{5}\left[\omega_{8}^{2} \omega^{5}\right]=0
$$

et cinq équations du troisiéme groupe

$$
\begin{aligned}
\left(\mathrm{V}_{3-7}\right)\left[\Delta l_{3 j}^{i}(\omega i]+\lambda_{3}\left[\omega_{9}^{j} \omega^{3}\right]\right. & =0 \\
(j & =1,2,4,5,6)
\end{aligned}
$$

que nous venons annexer aux equations $\left(\mathrm{V}_{1,2}\right)$.

Puis la troisième équation $(2: 5)$

$$
\omega_{9}^{10}=a_{9}^{10} \omega^{3}
$$

On obtient par une dérivation extérieure

$$
\left(\mathrm{III}_{11}\right) \quad\left[\Delta a_{9}^{10} \omega^{3}\right]=0
$$

où

$$
\Delta a_{9}^{10}=d a_{9}^{10}-\alpha_{9}^{10}\left(\omega_{3}^{3}+a_{9}^{9} \omega^{3}-\sum_{k \neq 0}^{k=1 \ldots 6} l_{k s}^{3} \omega^{k}\right) .
$$

Il nous reste à différencier les équations $(5: 4)$, (5:7) et (4:4); elles donnent

$$
\left(\mathrm{III}_{1-10}\right) \quad\left[\Delta a_{\frac{1}{j} \omega^{j}}=0, \quad(j=1, \ldots, 6)\right.
$$

$(5: 14)$

$$
\left[\Delta a_{8}^{j} \omega^{j}=0,(j=1,3,4,6)\right.
$$

où

$$
\Delta a_{7}^{j}=d a_{7}^{j}+a_{8}^{j=1 \ldots 6} \sum_{k \neq j}^{\Sigma} l_{k j}^{j} \omega^{k}-\sum_{k \neq j}^{\Sigma} l_{k j}^{j} \omega_{7}^{k},(j=1 \ldots 6)
$$

$(5: 15)$

$$
\Delta a_{8}^{j}=d a_{8}^{j}+a_{8}^{j} \sum_{k=1}^{k=1} l_{k j}^{i}\left(\omega^{k}-\sum_{k \neq j}^{k=1 \ldots 6} l_{k j}^{j} \omega_{8}^{k}, \quad(j=1,3,4,6)\right.
$$

et

$(5: 16)$

$$
\left(V_{1,2}\right) \quad\left[\Delta a_{9}^{7} \omega^{3}\right]=\lambda_{1}\left[\omega_{9}^{1} \omega^{1}\right]+\lambda_{4}\left[\omega_{9}^{4} \omega^{4}\right]+\lambda_{6}\left[\omega_{9}^{6} \omega^{6}\right]
$$

$$
\left[\Delta \alpha_{9}^{8} \omega^{3}\right]=\lambda_{2}\left[\omega_{9}^{2} \omega^{2}\right]+\lambda_{5}\left[\omega_{9}^{5} \omega^{5}\right]
$$


où

$$
\Delta a_{9}^{7}=d a_{9}^{7}-a_{9}^{7} \omega_{3}^{3}+\sum^{i=1,4,6} l_{i 3}^{3} \omega^{i}
$$

(5: 17$)$

$$
\Delta a_{9}^{8}=d a_{9}^{8}-a_{9}^{8} \omega_{3}^{3}+\stackrel{i=2,5}{\Sigma} l_{i 3}^{3} \omega^{i}
$$

§ 6. Le système des équations différentielles, qui détermine notre surface de Voss, coutient 65 équations de PFAfF, à savoir; $(1: 10,11),(2: 2,5)$, (3:4), 4: 4, 6, 7, 8). (5:4,7]. Les équations quadratiques sont réunies en cinq groupes, Le premier contient 6 équations. qui proviennent de la différenciation du système (1:10). Chaque équation du groupe contient un seul produit exterieur et un seul différentiel $d \lambda_{i}$

$$
\left(\mathrm{I}_{1-6}\right) \quad\left[\Delta \lambda_{i} \omega^{i}\right]=0
$$

Le second groupe contient 24 équations qui proviennent de la différanciation exterieure des équations $(3: 4)$. La sybstitution $(5: 4)$ on $(5: 7)$ les réduit à un seul terme

$$
\left(\mathrm{II}_{1-15}\right) \quad\left[\nabla l_{i j}^{j} \omega_{j}\right]=0
$$

$$
\left(\mathrm{II}_{16-3 \downarrow}\right) \quad\left[\nabla l_{2 j}^{j} \omega^{j}\right]=0,\left[\nabla l_{j 5}^{i} \omega^{j}\right]=0, \quad(j=1,3,4,6)
$$

Le troisième groupe contient onze équations, chacune à un terme, qui proviennent de la différenciation des équations (5:4), (5:7), (2:5).

$$
\left(\mathrm{III}_{1-11}\left\{\begin{array}{l}
{\left[\Delta a_{7}^{j} \omega^{j}\right]=0 \quad(j=1 \ldots)} \\
{\left[\Delta a_{8}^{j} \omega^{j}\right]=0 \quad(j=1,3,4,6)} \\
{\left[\Delta a_{9}^{10} \omega^{3}\right]=0 .}
\end{array}\right\}\right.
$$

Enfin, le cinquième groupe contient les dernières équations (3:4). Ce groupe contient les formes caractéristiques $\omega_{9}^{j}$.

$$
\left\{\begin{aligned}
\left(V_{1,2}\right)\left[\Delta \alpha_{9}^{7} \omega^{3}\right] & =\lambda_{1}\left[\omega_{9}^{1} \omega^{1}\right]+\lambda_{4}\left[\omega_{9}^{4} \omega^{4}\right]+\lambda_{6}\left[\omega_{9}^{8} \omega^{6}\right] \\
{\left[\Delta \alpha_{9}^{8} \omega^{8}\right] } & =\lambda_{2}\left[\omega_{9}^{2} \omega^{2}\right]+\lambda_{5}\left[\omega_{9}^{5} \omega^{5}\right]
\end{aligned}\right.
$$

$$
\left(\mathrm{V}_{3-7}\right)\left[\Delta l_{3 j}^{j} \omega^{j}\right]+3\left[\omega_{9}^{j} \omega^{3}\right]=0 \quad(j=1,2,4,5,6)
$$


chaque groupe des équations, au delà des formes $\omega^{i}$ indépendantes sur la variété intégrale, posséde ses formes du système caractéristique, diffèrentes des formes des autres groupes. Il en suit qu' on peut étudier chaque groupe à part. Si chaque sous-gronpe du système des équations quadratiques est involutive, le systeme en est également et les caractères sont égaux aux sommes des caractères correspondants des sous-groupes.

Les groupes I, II, III contiennent 41 equations, dont chaeune est à un seul terme. Le sous-système èvidemment est involutive. Le premier caractère est égal au nombre des équations, les autres sont nul

$$
\sigma_{1}=41, \sigma_{2}=\ldots=\sigma_{6}=0
$$

La quatriéme groupe contient deux équations et $q=4$ formes caractéristiques (au dela des $\left.\omega^{i}\right) \omega_{8}^{2}, \omega_{8}^{5}, \Delta l_{2 \tilde{n}}^{5}, \Delta l_{52}^{2}$. En appliquant le lemme de Cartan aux équations (6.6), on obtient $\mathscr{T}=6$ paramètres arbitraires. Les caractères du sous-système sont

$$
\sigma_{1}^{\prime}=2, \quad \sigma_{2}^{\prime}=2, \quad \sigma_{3}^{\prime}=0,=\ldots=\sigma_{k}^{\prime}=0
$$

Le nombre de CaRTan

$$
Q^{\prime}=\sigma_{1}^{\prime}+2 \sigma_{2}^{\prime}=6
$$

coincide avec $\mathscr{G} \mathcal{Z}^{\prime}$; le sous-système est involutive.

Il cinquième groupe contient 7 équations et $q=12$ formes caractéristiques, (au dela des $\omega^{t}$ ). En appliquant le lemme de CarTax aux équations $\left(V_{3-7}\right)$, on obtient

$$
\Delta l_{3 j}^{i}=a_{j} \omega^{j}+b_{j} \omega^{3}, \quad(j=1,2,4,5,6)
$$

$$
\lambda_{3} \omega_{9}^{j}=b_{j} \omega^{j}+c_{j} \omega^{3} .
$$

qui contient 15 paramètres arbitraires $a_{j}, b_{i}, c_{j}$. En portant les expressions des $\omega_{g}^{j}$ dans les equations $\left(V_{1,2}\right)$ on obtient

$$
\begin{aligned}
& \lambda_{3} \Delta a_{9}^{7}=\lambda_{3} a^{7} \omega^{3} c_{1} \lambda_{1} \omega^{1}-c^{4} \lambda_{4} \omega^{4}-c_{6} \lambda_{6} \omega^{6}, \\
& \lambda_{3} \Delta a_{9}^{8}=\lambda_{3} a^{3} \omega^{3}-c_{2} \lambda_{2} \omega^{2}-c_{5} \lambda_{5} \omega^{5}
\end{aligned}
$$

c' est encore deux paramètres arbitraires $a^{7}, a^{8}$; done en tout il existe $\mathscr{O}^{\prime \prime}=16$ paramètres arbitraires. Les caractères $d u$ sous-système sont

$$
\sigma_{1}^{\prime \prime}=7, \sigma_{2}^{\prime \prime}=5, \quad \sigma_{2}^{\prime \prime}=\ldots=\sigma_{6}^{\prime \prime}=0
$$


Le nombre de CARTAN

$$
Q^{\prime \prime}=7+2.5=17=N^{\prime \prime},
$$

donc le sous-système est involutive et tout le système en est égalemement avec les caractères

$$
S_{1}=\sigma_{1}+\sigma_{1}^{\prime}+\sigma_{1}^{\prime \prime}=50, S_{2}=\sigma_{2}^{\prime}+\sigma_{2}^{\prime \prime}=7, S_{2}=\ldots=S_{6}=0 .
$$

Il en suit que la variété de Voss en question dépend de 7 fonctions arbitraire des deux arguments.

§. En transformant les équations quadratiques nous avons souvent appliqué le prolongement partiel du système. Done il faut démontrer que le système charactéristique du système prolongé est complétement intégrable. Or e'est presque évident, toutes les équations de PFAFF ont été différenciées et les resultats ont été annexés au système. It reste à vérifier les formes

$$
\omega_{8}^{2}, \quad \omega_{8}^{5}, \omega_{9}^{i}(i=1,2,4,5,6), \Delta \lambda_{i}, \nabla l_{i j}, \Delta a_{7}^{i}(6, j=i \neq j)
$$

$$
\Delta a_{8}^{j}(j=1,3,4,6), \Delta a_{9}^{8}, \Delta a_{9}^{10},
$$

Or

$$
D \omega_{8}^{2} \equiv D \omega_{8}^{5} \equiv D \omega_{9}^{i} \equiv 0\left(\bmod \omega^{i}, i=1 \ldots 6\right)
$$

en vertu des équations du système.

Puis

$$
\begin{aligned}
D \Delta \lambda_{i} & \equiv-\left[d \lambda_{i} 2 \omega_{i}^{i}\right]-2 \lambda_{i} \underset{k \neq i}{\Sigma}\left[\omega_{i}^{k} \omega_{k}^{i}\right]+\underset{k}{\Sigma}\left[d l_{k i}^{i} \omega^{i}\right]+\underset{k}{\Sigma} l_{k i}^{i}\left[\omega^{l} \omega_{l}^{k}\right] \\
& \equiv-\left[d \lambda_{i} \omega_{i}^{i}\right]\left(\bmod \omega^{i}\right)
\end{aligned}
$$

Or l'oquation (5:2) nous donne

done

$$
d \lambda_{i} \equiv 2 \lambda_{i} \omega_{i}^{i}
$$

$$
D \Delta \lambda_{i} \equiv 0 . \quad\left(\bmod \omega^{t}\right)
$$

De mème, on démontre la congruence à zóro des différentielles extérieures des autres formes $(7: 1)$.

$\S 8$. Le tableau des composants des déplacements infinitesimales du repère de la surface $\mathfrak{K}_{6}$ nous permet de faire quelques conclusions sur la géometrie de la surface. 
Par exemple, chacune des cinq premières lignes coordonnées est située dans un plan fixe: les lignes $\omega^{k}=\delta_{i}^{k}(i=1,4,6)$ sont situées dans les plans $\left(M, \overrightarrow{e_{i}}, \overrightarrow{e_{7}}\right)$, les lignes $\omega^{k}=\delta_{j}^{k}(j=2,5)$ dans les plans $\left(M, \overrightarrow{e_{j}}, \overrightarrow{e_{8}}\right)$.

Les surfaces à deux dimensions

$$
\omega^{1}=\omega^{8}=\omega^{4}=\omega^{6}=0
$$

sont situées dans les espaces à trois dimensions $\left(M, e_{2}, e_{5}, e_{8}\right)$.

Joute la surface $\mathfrak{T K}_{6}$ est une famille à un paramètre des sous-variétés de Voss $\mathscr{O K}_{5}$, determinées par l'équation $\omega^{3}=0$ completémènt intégrable.

§ 9. La variété de Voss $\mathscr{O K}_{6}$ admet une déformation conservant le réseau conjugué des géodesiques et le type projectif de $\mathfrak{T K}_{6}$.

Considérons un couple des variétés de Voss $\mathfrak{K}_{6}$, $\mathfrak{T K}_{6}^{*} \mathrm{du}$ même type projectif, applicables avec conservation du réseau de géodésiques. Les deux surfaces sont déterminées par les composants $\omega_{i}^{j}$, $\omega_{i}^{\alpha} \ldots$ et ${ }_{\omega_{i}^{j}}^{*} \omega_{i}^{*} \ldots$ qui vérifient le système de Pfaff $(1: 10,11),(2: 2,5),(3: 4),(4: 3,4,6,7,8)$, $(5: 4,7)$ et des équations quadratiques $(I-V)$.

Désignons les différences des éléments correspondants des deux surfaces

$$
\overline{\omega_{i}^{\alpha}}=\omega_{i}^{\alpha}-\omega_{i}^{\alpha}, \overline{\lambda_{i} \omega_{8}^{j}}=\bar{\lambda}_{i}^{*} \omega_{8}^{i}=\lambda_{i} \omega_{8}^{j}-\lambda_{i} \omega_{8}^{j} \lambda_{i} \omega_{8}^{j} \text { etc. }
$$

L'élément linéaire $\mathfrak{T K}_{6}$ est

$$
d s^{2}=d \vec{M}^{2}\left(\omega^{i} \overrightarrow{e_{i}}\right)^{2}={ }^{i=1} \Sigma^{6}\left(\omega^{i}\right)^{2}+2^{i, j=1 \ldots 6} \sum_{i \neq i}^{i \neq \ldots} P^{i j} \omega^{i} \omega^{i}
$$

où $p_{i j}=e_{i} e_{j}$ le cosinus de l'angle des vecteurs $e_{i}, e_{j}$ et est invariant; comme

$$
d p_{i j}=\omega_{i}^{k} p_{k j}+\omega_{j}^{k} p_{i k}
$$

les formes $\omega_{i}^{j}$ sont invariantes, et l'équation (3:4) montre que les coefficients $l_{i j}^{i}$ sont invariants egalement.

Les différences des équations de PFAFF correspondantes sont

$$
\begin{aligned}
& (9: 3) \quad \bar{\omega}_{i}^{7}=\bar{\lambda}_{i} \omega^{i},(i=1,4,6), \bar{\omega}_{j}^{8}=\bar{\lambda}_{j} \omega^{i}(j=2,5), \bar{\omega}_{\mathrm{B}}^{9}=\bar{\lambda}_{3} \omega^{3}, \\
& (9: 4) \quad \bar{\omega}_{i^{7}}^{7}=0\left(i^{\prime}=2,3,5\right), \bar{\omega}_{j^{\prime}}^{8}=0,\left(j^{\prime}=1,3,4,6\right), \bar{\omega}_{k}^{9}=0,\left(\begin{array}{c}
k=1 \\
k \neq 3
\end{array}, \ldots 6\right) \\
& (9: 5) \quad \bar{\omega}_{7}^{8}=\bar{\omega}_{7}^{9}=\bar{\omega}_{8}^{7}=\bar{\omega}_{8}^{9}=0, \bar{\omega}_{9}^{7}=\bar{a}_{9}^{7} \omega^{3}, \bar{\omega}_{9}^{8}=\bar{a}_{9}^{8} \omega^{3}, \\
& (9: 6) \quad \bar{\omega}_{7}^{i}=\bar{a}_{7}^{i} \omega^{1}(i=1 \ldots 6), \bar{\omega}_{8}^{j}=\bar{\alpha}_{8}^{j} \omega^{i}(j=1,3,4,6), \bar{\omega}_{9}^{10}=\bar{a}_{9}^{10} \omega^{3} .
\end{aligned}
$$


S. Finixof: Une surface de Voss à $\mathbf{6}$ dimensions dont le sous-espace, etc. 53

Les equations quadratique:

1. L'équation $\left(I_{1-6}\right)$ prend la forme

$(9: 7)$

$$
\left(\mathbf{I}_{\overline{1}-\overline{6})} \quad\left[\Delta \lambda_{i} \omega^{i}\right]=0\right.
$$

où

$$
\bar{\Delta} \lambda_{i}=\stackrel{*}{\Delta} \lambda_{i}-\Delta \lambda_{i}=\overline{d \lambda_{i}}-2 \bar{\lambda}_{i} \omega_{i}^{i}+\bar{\lambda}_{i}^{k=1 \ldots 6} \sum_{k \neq i}^{\Sigma} l_{k i}^{i}(i=1,2,4,6)
$$

$$
\overline{\Delta \lambda}_{3}=\bar{d}_{3}-2 \bar{\lambda}_{3} \omega_{3}^{3}+\bar{\lambda}_{3} a_{3}^{3} \omega^{3}+\bar{\lambda}_{3} \sum_{k \neq=1}^{k=6} l_{k 3}^{3}
$$

2. Les equations quadratiques du second groupe donnent des équations finies.

En vertu de (5:3) on a

$$
\overline{\Delta l} l_{i j}^{j}=0
$$

done l'équation (5: 6) donne

$$
\bar{\nabla} l_{i j}^{j}=-\overline{\lambda_{i} a_{7}^{j} \omega^{i}}\left(\begin{array}{l}
i=1,4,6 \\
j=1, \ldots 6, \neq i
\end{array}\right)
$$

Les équations $\left(\mathrm{II}_{1-15}\right)$ prennent la forme

$$
\overline{\lambda_{i}} \alpha\left[\omega_{7}^{i} \omega^{j}\right]=0
$$

Done

(9: 11)

$$
\left(\mathrm{II}_{\overline{1}} \ldots \overline{15}\right) \quad{\stackrel{*}{\lambda_{1}}}_{1}^{*} \frac{i}{7}-\lambda_{1} a_{7}^{j}=0 .
$$

De même, en vertu de $\left(\begin{array}{ll}5 & 9\end{array}\right)$

$$
\overline{\nabla l j}=-\overline{\lambda_{2} a_{8}^{j}} \omega^{2}, \quad \bar{\nabla} l_{5 j}^{i}=-\overline{\lambda_{5} a_{8}^{j}} \omega^{5}
$$

et le $\left(\mathrm{II}_{16-24}\right)$ donne

(9:13)

$$
\left(\mathrm{II}_{1 \overline{16}} \ldots \overline{24}\right) \quad{\stackrel{*}{\lambda_{2}}}_{2}^{*}{ }_{8}^{j}=\lambda_{2} a_{8}^{j}, \quad \stackrel{*}{\lambda_{5}} \stackrel{*}{a_{8}^{j}}=\lambda_{5} a_{8}^{j}
$$

3. Les equations $(5: 13)$, (5: 15) nous donnent

$$
\bar{\Delta} a_{7}^{j}=\bar{d} a_{7}^{j}+\bar{a} \frac{a_{7}^{j}}{k=1 \ldots 6} \sum_{k \neq j} l_{k j}^{j} \omega^{k}-\sum_{k \pm j}^{k=1 \ldots 6} l_{k_{j}}^{j} \bar{a}_{7}^{k}
$$




$$
\overline{\Delta a}_{8}^{j}=\overline{d a} a_{8}^{j}+\bar{a}_{8}^{j} \sum_{k \neq j}^{k=1 \ldots 6} l_{k j}^{j} \omega^{k}-\sum_{k \pm j}^{k=1,3_{1}, 6} l_{k j}^{j} \bar{a}_{3}^{k}
$$

(9: 15)

$$
\overline{\Delta a_{9}^{10}}=\overline{d a_{9}^{10}}-\overline{a_{9}^{10}} \omega_{8}^{3}-\overline{a_{9}^{10}} a_{9}^{9} \omega^{3}+\bar{a}_{9}^{10} \sum_{k \neq 0}^{k=1 \ldots 6} l_{k 3}^{3} \omega^{k}
$$

et le sous-système $\left(\mathrm{III}_{1-11}\right)$ donne

$$
\left[\overline{\Delta a} j_{7}^{j} \omega^{i}\right]=0 \quad(i=1 \ldots 6)
$$

$$
\left(\mathrm{III}_{\overline{1}} \ldots \overline{11}\right) \quad\left[\overline{\Delta a} \bar{s}_{g}^{j_{j} j}\right]=0 \quad(i=1,3,4,6)
$$

$$
\left[\bar{\Delta} \alpha_{9}^{10} \omega^{3}\right]=0
$$

4. Comme

$$
\overline{\Delta l}_{25}^{5}=0, \overline{\Delta l_{52}^{2}}=0
$$

le sous-système $\left(I V_{1,2}\right)$ prend la forme

$$
\left(I V_{\overline{1}}, \overline{2}\right) \quad\left[\overline{\lambda_{2} \omega_{8}^{5}}, \omega^{2}\right]=0,\left[\overline{\lambda_{5} \omega_{8}^{2}}, \omega^{5}\right]=0
$$

5. Les équations (5: 17) donnent

$$
\overline{\Delta a_{9}^{7}}=\overline{d a_{9}^{7}}-\bar{a}_{9}^{7} \omega_{3}^{3}, \quad \overline{\Delta a_{9}^{8}}=d \overline{a_{9}^{8}}-\overline{a_{9}^{8} \omega_{3}^{3}}
$$

et les équations $\left(\nabla_{\overline{1}}, \overline{2}\right)$ prennent la forme

$$
\left(V_{i, \overline{2}}\right) \quad\left\{\begin{array}{l}
\left.\left[\overline{\Delta a_{9}^{7} \omega^{8}}\right]=\left[\overline{\lambda_{1} \omega_{9}^{1}}, \omega^{1}\right]+\left[\overline{\lambda_{4} \omega_{9}^{4}}, \omega^{4}\right]+\overline{\lambda_{6} \omega_{9}^{6}}, \omega^{6}\right] \\
{\left[\overline{\left.\Delta a_{9}^{8} \omega^{3}\right]}\right]=\left[\overline{\lambda_{2} \omega_{9}^{2}}, \omega^{2}\right]+\left[\overline{\lambda_{5} \omega_{9}^{5}}, \omega^{5}\right]}
\end{array}\right.
$$

Enfin, en vertu de $\Delta_{3 j}^{j}=0$ les equations $\left(\nabla_{\overline{3}-\overline{7}}\right)$ prennent la forme

$$
\left(V_{\overline{3}} \ldots \overline{7}\right)\left[\overline{\lambda_{3} \omega_{9}^{j}}, \omega^{3}\right]=0 \quad(j=1,2,4,5,6)
$$

\$ 10. Etude des relations finis.

Groupe premier contient la relation $(9 ; 11)$

$$
\left(\mathrm{II}_{\overline{1}} \ldots \overline{15}\right) \quad \lambda_{i}^{*} a j=\lambda_{i} a \frac{j}{7}, \quad \begin{aligned}
& i=1,4,6 \\
& j=1 \ldots 6 j \neq i
\end{aligned}
$$


il en suit

$$
\frac{a^{*} \frac{j}{i}}{a i}=\frac{\lambda_{i}}{\lambda_{i}^{*}}=\varphi
$$

Comme les indices $i$ et $j$ prennent leurs valeurs indépendamment, le rapport $\mu$ de leurs grandeurs n' en dépend pas.

Done

$$
{\stackrel{*}{\lambda_{i}}}_{i}=\frac{1}{\varphi} \lambda_{i}, \stackrel{*}{a_{7}^{j}}=\varphi a_{7}^{j}\left(\begin{array}{l}
i=1,4,6 \\
j=1 \ldots 6
\end{array}\right)
$$

En différenciant les logarithmes de chaque membre des egalités (10:1), on obtient en vertu de

$$
\begin{gathered}
\frac{\Delta \lambda_{i}^{*}}{\lambda_{i}^{*}}-\frac{\Delta \lambda_{i}}{\lambda_{i}}+d \text { en } \varphi=0 \\
\frac{\Delta a \frac{j}{7}}{* \frac{*}{a j}}-\frac{\Delta a a_{7}^{j}}{a_{7}^{j}}=\sum_{k=1}^{k=1} l_{k j}^{j} l_{k j}^{j}\left(\frac{a_{7}^{k}}{\frac{*}{a}}-\frac{a_{7}^{k}}{a_{7}^{j}}\right) \omega^{k}+d \text { en } \varphi
\end{gathered}
$$

Ici en vertu de (10:1) la parenthèse devient nul; comme en vertu de de $\left(\mathrm{I}_{1-6}\right),\left(\mathrm{I}_{\overline{1}-6}^{-6}\right), \Delta \lambda_{i}, \Delta \stackrel{\ddot{\lambda}_{i}}{ }$ ne dépendent que de $\omega^{i}$ et en vertu de $\left(\mathrm{III}_{1-10}\right)$, $\left(\mathrm{III}_{\overline{1}} \ldots \overline{\mathrm{10}}\right)$ ne dépendent que de $\omega^{i}$, la différentielle $d$ en $\varphi$ est égale à zero.

Ainsi

$$
\varphi=\text { const, } \quad \Delta \stackrel{*}{*} \lambda_{i}=\frac{1}{\varphi} \Delta_{i}, \quad \Delta \stackrel{*}{j}=\varphi a_{7}^{j}
$$

et les équations $\left(\mathrm{I}_{\overline{1}}, \overline{4}, \overline{6}\right)$ et $\left(1 \mathrm{II}_{\overline{1}}, \ldots \overline{11}\right)$ sont vérifiées.

Le second groupe contient les relations (9:13).

$$
\left(\mathrm{II}_{\overline{16}-\overline{24})} \begin{array}{l}
{\stackrel{\lambda_{2}}{*} a_{8}^{j}}^{*}=\lambda_{2} a_{8}^{j} \\
\vec{\lambda}_{5}^{*} a_{9}^{j}=\lambda_{5} a j
\end{array}(j=1,3,4,6)\right.
$$

II en suit

$$
\frac{\stackrel{*}{a_{8}^{j}}}{a_{8}^{j}}=\frac{\lambda_{2}}{\lambda_{2}^{*}}=\frac{\lambda_{5}}{\lambda_{5}^{*}}=\psi
$$


ou

$(10: 3)$

$$
\lambda_{i}=\frac{1}{\psi} \lambda_{i}(i=2,5) \quad \stackrel{*}{a j}=\psi a_{8}^{j}(j=1,3,4,6)
$$

En dérivant logarithmiquement deux membres de la première équation, on obtient

$$
\psi=\text { const } \Delta \lambda_{i}^{*}=\frac{1}{\psi} \Delta \lambda_{i} \quad(i=2.5)
$$

La seconde équation à l'aide de (5: 15) donne

$$
\left(\frac{\Delta a_{8}^{j}}{\psi}-\Delta a_{8}^{j}\right)+l_{2 j}^{\dot{j}}\left(\frac{\omega_{8}^{2}}{\psi}-\omega_{8}^{2}\right)+l_{5 j}^{j}\left(\frac{\omega_{8}^{5}}{\psi}-\omega_{8}^{5}\right)=0 .
$$

Ici la première parenthèse en vertu de $\left(\operatorname{III}_{1-10}\right)$ ne dépend que de $\omega^{j}(j=1,3,4,6)$; tandis que en verta de $\left(\mathrm{IV}_{\overline{\mathrm{i}}}, \overline{2}\right)$ en transformant à l'aide de la première équation (10 3), on obtient

$$
\left[\frac{\omega_{8}^{5}}{\psi}-\omega_{8}^{5}, \omega^{2}\right]=0,\left[\frac{\omega_{8}^{2}}{\psi}-\omega_{8}^{2}, \omega^{5}\right]=0 .
$$

Ainsi la seconde et la troisième paranthèses $(10: 5)$ ne dèpendent que de $\omega^{5}$ et de $\omega^{2}$ conformement. Donc, chachune de trois parenthèses est égale à zéro

$(10: 6)$

$$
\Delta \stackrel{*}{a}=\psi \Delta a_{8}^{j} \quad(j=1,3,4,6
$$

$$
\stackrel{*}{\omega}_{8}^{2}=\psi \omega_{8}^{2}, \quad \stackrel{*}{*}^{5}=\psi \omega_{8}^{5}
$$

L'équation $(10: 6)$ et la seconde de $10: 4)$ se vérifient identiquement en vertu de 10:3) et $\psi=$ const; elles reduisent à l'identité $\left(\mathrm{I}_{\overline{2}}, \overline{\bar{\sigma}}\right)$ et $\left(\mathrm{III}_{\bar{\gamma}}-\overline{10}\right)$.

Les équations $(10: 7)$ sont complétement intégrables et vérifient les équations quaratiques $\left(\mathrm{IV}_{\overrightarrow{1}}, \vec{z}\right)$.

\section{§11. Le théorème d'existence.}

Ainsi, au-delà des équations finies

$$
\begin{aligned}
& \dot{\lambda}_{i}^{*}=\frac{1}{\varphi} \lambda_{i}(i=1,4,6), \quad \lambda_{j}^{*}=\frac{1}{\psi} \lambda_{j}(j=2,5) \\
& \stackrel{*}{a j}=\varphi a_{7}^{i}(j=1 \ldots 6), \quad \stackrel{*}{a} \frac{i}{7}=\psi a_{8}^{i}(j=1,3,4,5)
\end{aligned}
$$


et une série des équations de PFAFF, y compris les équations complétement intégrables $(10: 7)$, il reste pour déterminer la déformation $\mathfrak{O K}_{6}^{*}$ neuf équations quadratiques

$$
\begin{aligned}
& \left(\mathrm{III}_{\overline{11}}\right) \quad\left[{\overline{\Delta \lambda_{3}}}_{3}^{3}\right]=0\left(\mathrm{I}_{3}\right),\left[\overline{\Delta a}_{9}^{10} \omega^{8}\right]=0 \\
& \left(\mathrm{~V}_{\overline{1}, \overline{2}}\right)\left\{\begin{array}{l}
{\left[\overline{\left.\Delta a_{9}^{7} \omega^{3}\right]}=\left[\overline{\lambda_{1} \omega_{9}^{1}}, \omega^{1}\right]+\left[\overline{\lambda^{4} \omega_{9}^{4} \omega^{4}}\right]+\left[\overline{\lambda_{6} \omega_{9}^{6}}, \omega^{6}\right]\right.} \\
{\left[\overline{\left.\Delta a_{9}^{8} \omega^{3}\right]}=\left[\overline{\lambda_{2} \omega_{9}^{2}}, \omega^{2}\right]+\left[\overline{\lambda_{5} \omega_{9}^{5}}, \omega^{5}\right]\right.}
\end{array}\right. \\
& \left(\nabla_{\overline{3}} \ldots \overline{7}\right) \quad\left[\bar{\lambda}_{3\left(\omega_{9}^{j}\right.}^{j} \omega^{3}\right]=0 \quad(j=1.2 .4 .5 .6) .
\end{aligned}
$$

11 est évident que les deux premières équations sont involutives. Le système $\left(\nabla_{\overline{1}} \ldots \overline{\overline{7}}\right)$ contient $\sigma_{1}=7$ equations quadratiques et 9 formes caractéristiques $\overline{\Delta a_{9}^{7}}, \overline{\Delta a}_{8}^{9}$ et $\bar{\omega}_{9}^{j}(j=1$. 2. 4. 5. 6). En appliquant le lemme de CaRTAN on obtient

$$
\lambda_{3 \omega_{9}^{j}}^{*}=\lambda_{3 \omega_{9}^{j}}+\stackrel{*}{a_{9}^{j} \omega^{3}}
$$

Comme la première variété est donnée, les formes $m_{9}^{j}$ sont connues; on peut les prendre de la seconde équation $(6: 10)$

$$
\lambda_{3} \omega_{9}^{j}=b_{j \omega} \omega^{j}+c_{j} \omega^{3}
$$

En portant l'expression

$$
\dot{\lambda}_{3}^{*} \omega_{9}^{j}=b_{j} \omega^{j}+\left(\stackrel{*}{a}_{y}^{j}+c_{j}\right) w^{3} \quad(j=1.2 .4 .5 .6)
$$

dans les équations $\left(\mathrm{V}_{\overline{1}, \overline{2}}\right)$, on obtient

$$
\begin{aligned}
& \left.\dot{\nu}_{3}^{*} \overline{\Delta a_{9}^{7}}=-\left(\dot{\lambda}_{1} a_{9}^{1}+\ddot{\lambda}_{[1}^{*} \lambda_{3]}\right) \omega^{\prime}-\ddot{(}_{\lambda_{1}}^{*}{ }^{*} a_{9}^{4}+\lambda_{[4} \lambda_{3]}\right) \omega^{4}- \\
& -\left(\lambda_{6}^{*} a_{9}^{6}+\lambda_{[6}^{*} \lambda_{3]}\right) \omega^{6}+{ }^{*} a_{9}^{7} \omega^{3} \\
& \left.\lambda_{3}^{*} \Delta \alpha_{9}^{8}=-\left(\lambda_{2}^{*} a_{9}^{*}+\lambda_{[2}^{*} \lambda_{3}\right)\right)^{2}-\left(\lambda_{5}{ }^{*} a_{9}^{5}+\lambda_{[5}^{*} \lambda_{3}\right) \omega^{3}+ \\
& +a_{9}^{8} \omega^{3}
\end{aligned}
$$

Les développements $(11: 2,3)$ contiennent $N=7$ paramètres arbitraires

$$
\stackrel{*}{a_{0}^{7}}, \stackrel{*}{a_{9}^{8}} \text { et } \stackrel{*}{a_{8}^{j}}(j=1.2 .4 .5 .6)
$$


Comme les caractères du sous-système $\left(\mathrm{V}_{\overline{1}} \ldots \overline{\overline{7}}\right)$ sont

$$
\sigma_{1}=7, \quad \sigma_{2}=\ldots=\sigma_{6}=0 .
$$

Le nombre de CARTAN $Q=7=N$; le sous-système est involutive et tout le système egalement avec les caractères

$$
S_{1}=9, \quad S_{2}=\ldots=S_{6}=0 .
$$

Ainsi la surface de Voss $\mathfrak{O K}_{6}$ du type projectif consideré admet la déformation continue en surface de Voss de même type avec neuf fonctions arbitraires d'un argument.

Il est remarquable, que ces neuf fonctions arbitraires sont liees à la forme $\omega^{3}$. Si l'on pose

$$
\omega^{3}=0
$$

c.à.d. si l'on considère une sous-variété $\mathfrak{O K}_{5}$ de la famille à un paramètre des variétés $\left(\mathscr{O K}_{\overline{5}}\right)$ qui compose la variété $\mathscr{T K}_{6}$, les équations ( $\left.\mathrm{I}_{\overline{3}}\right)$, (IIII $\left.\bar{I}_{11}\right)$, $\left(V_{\overline{1}-\overline{7}}\right)$ disparaissent. Chaque $\mathscr{O}_{5}$ est située dans un $\mathscr{G}_{7}$ euclidien déterminé par les vecteurs $\vec{e}_{i}, \vec{e}_{\alpha}(i \neq 3, \alpha \neq 9)$. La déformation de la sous-variété $\mathfrak{O K}_{\tilde{n}}$, forcée par la déformation de $\mathfrak{O K}_{6}$ est dégénérée en l'extension des vecteurs $\overrightarrow{e_{7}}, \overrightarrow{e_{8}}$ en $\varphi$ et $\psi$ fois conformemant, c. à.d. en transformation centro affine de l'espace $\mathscr{G}_{7}$. 